\title{
Editorial: Digitization of Organizations: Towards a New Paradigm of Real-Time Systems
}

\author{
Igor Litvinchev ${ }^{1} \cdot$ Tatiana Romanova ${ }^{2,3}$
}

Published online: 5 February 2022

(c) The Author(s), under exclusive licence to Springer Science+Business Media, LLC, part of Springer Nature 2022

\section{Contents}

Digital twin large-scale optimization problems in supply chains: A case of packing problem.

Jose Antonio Marmolejo-Saucedo

Microdata analytics of out-of-pocket and catastrophic health spending in Mexico: an analysis by quantiles.

Roman Rodriguez-Aguilar

Demand forecasting for freight transport applying machine learning into the logistic distribution.

Jania Astrid Saucedo-Martinez

\section{Editorial}

The success of any company depends, among other things, on the ability to use resources efficiently. Today, organizations experience a process called digital transformation. Through this process companies make use of technology to improve performance, increase income and ensure better results. Its implementation, however, requires structural changes in organizations according to the objectives they want to achieve.

Disruptive technologies allow companies to change completely their reality, from the experience they offer to their customers to the way they develop their internal processes and even the organizational culture they live daily. All agents involved in the business must participate in the

Igor Litvinchev

igorlitvinchev@gmail.com

1 Nuevo Leon State University (UANL), San Nicolás de los Garza, Nuevo Leon, Mexico

2 Kharkiv National University of Radio Electronics (NURE), Kharkiv, Ukraine

3 Institute for Mechanical Engineering Problems of the National Academy of Sciences of Ukraine, Kharkiv, Ukraine digital transformation process, from planning to effective implementation.

In recent years, the concept of Digital Twins has been developed. This concept is a digital representation of a physical asset, process, or a system, as well as the engineering information that allows understanding and modeling the performance. The Digital Twin can be continuously updated using, e.g., sensors and continuous inspections, to represent its status, function, or position in nearly real time. The Digital Twin enables users to view assets, check their status, perform analysis, and generate information to predict and optimize their performance.

Modeling, simulation, and optimization of Digital Twins requires application of cutting-edge techniques from mathematical programming, data analytics and cloud computing. Only this way it is possible creating digital twins providing accurate simulations of current, past, and future operations.

This special issue features three selected papers. The first article, "Digital twin framework for large-scale optimization problems in supply chains: A case of packing problem" by J.A. Marmolejo-Saucedo, presents the nested solution of two large-scale optimization problems, vehicle routing and bin packing. The technological platform is based on a simulation engine that is linked to an interface to coordinate various interconnections in a company's supply chain.

In the second article, "Microdata analytics of out-ofpocket and catastrophic health spending in Mexico: an analysis by quantiles" by R. Rodriguez-Aguilar, the design of public policies for health systems is presented. The approach is based on digitalizing information from a user registry and allows generating sufficient public financing policies to avoid catastrophic expenses for the population. A statistical analysis in real time is proposed to monitor day by day changes in the budget requirements for the care of various diseases.

In the last paper, "Demand forecasting for freight transport applying machine learning into the logistic distribution", J.A. Saucedo-Martinez proposes a methodology to 
compare three prediction methods: traditional statistical method, hybrid method and artificial neural network. The methods are tested by the Mean Squared Error indicator to select the approach providing more accurate results in the forecast of the demand for freight transport. Experimental results demonstrate that the historical data saved in a transport management system can be used for reliable freight transportation demand forecasting.

Acknowledgement We would like to express our deep thanks to the Editor-in-Chief, Professor Imrich Chlamtac for providing us with the opportunity to host this special issue and for his supportive guidance during the entire process. We also thank the authors for submitting the papers and all reviewers for providing their evaluations and recommendations.

Publisher's Note Springer Nature remains neutral with regard to jurisdictional claims in published maps and institutional affiliations.

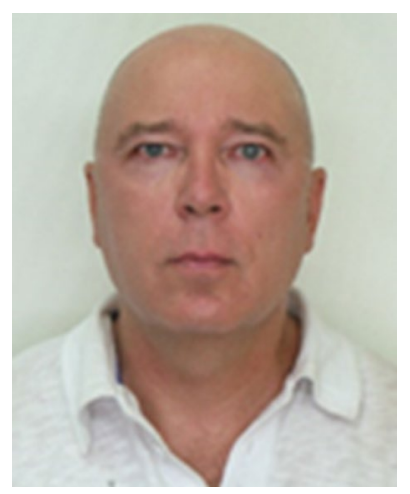

Igor Litvinchev received his M.Sc. degree in Applied Mathematics from Moscow Institute of Physics and Technology (Fizteh), Russia; Ph.D. in Systems Theory and Operations Research and Dr.Sci. (Habilitation) in Systems Modeling and Optimization, both from Computing Center, Russian Academy of Sciences (CCAS), Moscow. He is currently a Professor at Nuevo Leon State University (UANL), Mexico and a Leading
Mathematician at CCAS. His research is focused on large-scale systems modeling, optimization, and control with applications to interdisciplinary research. He is an author of 4 books and an editor of 6 more. He published more than 90 research papers in leading international journals and served in Program and Organizing Committees for 47 international conferences. His research was supported by 31 grants from NATO Scientific Affairs Division and European Community; ISF (USA) and RFBR (Russia); CNPq and FAPESP (Brasil); BRFBR (Belarus); CONACYT, PROMEP and PAICYT (Mexico). Litvinchev is a member of Russian Academy of Natural Sciences and Mexican Academy of Sciences.

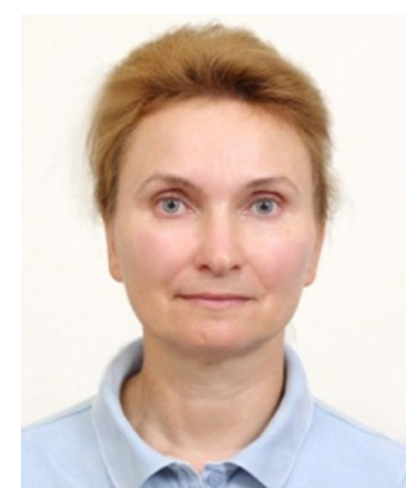

Tatiana Romanova received his M.Sc. degree in Applied Mathematics from Kharkiv National University of Radio Electronics (NURE), Ukraine; Ph.D. and Dr.Sci. (Habilitation) in Mathematical Modeling and Computational Methods from the Institute of Cybernetics of the National Academy of Sciences of Ukraine, Kiev. She is a leading researcher of the Department of Mathematical Modeling and Optimal Design at the Institute for Mechanical Engineering Problems of the National Academy of Sciences of Ukraine in Kharkiv and professor at the chair of Applied Mathematics at the Kharkiv National University of Radioelectronics. She is an author of numerous research papers focused on the development of geometric tools, mathematical models and optimization algorithms for solving packing, cutting, covering and layout problems. Her research was supported by grants from Deutsche Forschungsgemeinschaft (GIO), STCU, Volkswagen, FRFU (Fundamental Research Foundation of Ukraine). She was invited to give lectures on the phifunction technique at universities of Dresden (Germany), Southampton (England), Cardiff (Wales), Porto(Portugal), Monterrey, Mexico (Mexico). She is a member of ESICUP, EWG, EURO, OPTISAD. 